\title{
On the central role of brain connectivity in neurodegenerative disease progression
}

\author{
Yasser Iturria-Medina ${ }^{1,2 *}$ and Alan C. Evans ${ }^{1,2}$ \\ ${ }^{1}$ Montreal Neurological Institute, Montreal, QC, Canada, ${ }^{2}$ Ludmer Center for Neurolnformatics and Mental Health, Montreal, \\ QC, Canada
}

Increased brain connectivity, in all its variants, is often considered an evolutionary advantage by mediating complex sensorimotor function and higher cognitive faculties. Interaction among components at all spatial scales, including genes, proteins, neurons, local neuronal circuits and macroscopic brain regions, are indispensable for such vital functions. However, a growing body of evidence suggests that, from the microscopic to the macroscopic levels, such connections might also be a conduit for in intra-brain disease spreading. For instance, cell-to-cell misfolded proteins (MP) transmission and neuronal toxicity are prominent connectivity-mediated factors in aging and neurodegeneration. This article offers an overview of connectivity dysfunctions associated with neurodegeneration, with a specific focus on how these may be central to both normal aging and the neuropathologic degenerative progression.

OPEN ACCESS

Edited by:

Manuel Menéndez-González, Hospital Álvarez-Buylla, Spain

Reviewed by: J. Arturo García-Horsman, University of Helsinki, Finland Rubem C. A. Guedes, Universidade Federal de Pernambuco, Brazi

*Correspondence:

Yasser Iturria-Medina, Montreal Neurological Institute, 3801 University Street, room NW145, Montreal, QC H3A 2B4, Canada iturria.medina@gmail.com

Received: 16 March 2015 Accepted: 01 May 2015 Published: 21 May 2015

Citation: Iturria-Medina Y and Evans AC (2015) On the central role of brain connectivity in neurodegenerative disease progression

Front. Aging Neurosci. 7:90. doi: 10.3389/fnagi.2015.00090
Keywords: brain connectivity, deregulated gene networks, misfolded proteins, neuronal activity toxicity, metabolic dysfunction, vascular deregulation, disease spreading, neurodegeneration

\section{Introduction}

Intra-brain connectivity is indispensable for the attainment and maintenance of animal life. Genes, proteins, neurons, cell assemblies and gross brain regions all interact constantly to orchestrate the brain functions that underly sensorimotor and cognitive processing. Homeostatic mechanisms establish a basal level of functional organization upon which subtle variations are overlaid that subserve the changes in mood, attention, performance and response to external stimuli that we discern at the behavioral level. However, this delicate equilibrium may break down, particularly in the presence of neurological and psychiatric disorders where aberrant pathologic factors provoke massive alterations in connectivity at all brain levels (Konrad and Eickhoff, 2010; Bicchi et al., 2013; Iturria-Medina, 2013; Reynolds and Stewart, 2013; GomezRamirez and $\mathrm{Wu}, 2014$; He and Evans, 2014; Pievani et al., 2014). Recent advances in brain mapping tools, including genetics, electrophysiology and imaging techniques, with the support of new bioinformatic analysis, have extended to unprecedented levels our understanding of segregation and integration processes in the normal brain (Stam and van Dijk, 2002; Bota et al., 2003; Hagmann et al., 2007; Iturria-Medina et al., 2007; Karlebach and Shamir, 2008; Axer et al., 2011; Friston, 2011; Sporns, 2011; Evans, 2013). Additionally, they have revealed the connectional alterations associated with a wide range of psychiatric and neurological disorders (Buckholtz and Meyer-Lindenberg, 2012; Meyer-Lindenberg and Tost, 2012). Both brain disconnections and hyperconnections are commonly observed for different neurodegenerative diseases (for detailed review see Pievani et al., 2014). How disconnections and hyperconnections arise and coexist during disease progression is still not well understood. Often, disconnections 
are considered a direct consequence of neurodegeneration, while hyperconnections are assumed to reflect compensatory mechanisms or spatiotemporal correlation in pathology. But such views may be a simplistic interpretation of more complex phenomena, in which brain connectivity could be playing a more causal role. In this article, we provide a brief overview of the biological mechanisms implicated in connectional dysfunctions and consequent neurodegeneration. The article is organized in four primary subsections. The first offers a brief overview of gene regulatory network alterations and their role in neurodegeneration. The second reviews the demonstrated role of the brain's structural architecture on prion-like propagation, as a main factor mediating neurodegenerative progression. The third presents and discusses the evidence supporting the neuronal activity dependent neurodegeneration hypothesis, in which functional connectivity presents an active role. The fourth integrates previous and recent findings, emphasizing the role of multimodal connections on disease spreading and progression. Finally, we highlight some outstanding questions and the challenges in building an operational model of dynamic brain organization that can account for both normal brain aging and neurodegenerative disease.

\section{Alterations in Normal Brain Connectivity and Neurodegeneration}

\section{Deregulated Gene and Protein Networks}

Gene regulatory networks control the expression levels of mRNA and proteins. Normal cellular activity depends upon the proper functioning of these networks. This makes the analysis of regulatory network dynamics a crucial step towards understanding the biological processes of health and disease (for reviews, see Bota et al., 2003; Karlebach and Shamir, 2008; Bernot et al., 2013). Aging and neurodegeneration are thought to have strong upstream genetic causes. For instance, Apoe\&4 and BCHE genes are considered important risk factors for the development of Alzheimer's disease (AD; Genin et al., 2011; Cramer et al., 2012; Ramanan et al., 2014). Meanwhile, increasing evidence supports the important modulatory impact of many other ADrelated genes (Lambert et al., 2013). Similarly, amyotrophic lateral sclerosis (ALS) is associated with different genetic risk factors, e.g., TDP-43 and SOD, which act in combination with aging and environmental conditions (for reviews see Al-Chalabi and Hardiman, 2013; Robberecht and Philips, 2013). Such multifactorial causes during the neuropathologic progression are common for the most prevalent neurodegenerative diseases of AD, ALS, Frontotemporal dementia (FTD), Parkinson's disease (PD) and Huntington's disease (HD). It strongly supports that "aberrant" genes do not act alone on pathologic progression but by their interaction with other collaborator genes under the influence of environmental/experience conditions (e.g., life style, epigenetic effects). Nutrition conditions have an important modulatory role on gene activities and aging disorders (Joseph et al., 2009; Bouchard-Mercier et al., 2013; Nicolia et al., 2014). For instance, caloric restriction and diet rich in anti-inflammatory and antioxidant properties have been found associated to increased longevity and preserved cognitive functioning (Roth et al., 2002; Colman et al., 2009; Joseph et al., 2009; Stice et al., 2013; Crichton et al., 2013; Sezgin and Dincer, 2014). Nutri-epigenomics science focus on the influence of nutrition on epigenetic modifications and its consequences on health (Gallou-Kabani et al., 2007), which ideally should contribute to develop effective nutrition-based therapeutic interventions. Modern gene expression profiling techniques allow us to quantify gene-specific activity across different tissues and time points (O'Driscoll, 2011). As a result, genetic interaction occurring between regions of interest can be characterized by means of sophisticated statistical concepts and tools (Bota et al., 2003; Karlebach and Shamir, 2008; Bernot et al., 2013), thereby contributing to our understanding of how regulatory networks are involved in disease progression (Crespo et al., 2012; Narayanan et al., 2014; Leiserson et al., 2015). In the context of brain degeneration, Zhang et al., 2013, reported a remarkable example of causal gene-gene pathologic interaction. These authors used gene expression profiles of the prefrontal cortex to identify regulatory networks causally associated with late onset $\mathrm{AD}$. They identified an immune and microgliaspecific gene module that is strongly regulated by the gene TYROBP, directly associated to Amyloid- $\beta$ (A $\beta$ ) turnover and neuronal damage. This TYROBP causal network (Figure 1A), characterized in detail by means of Bayesian network analysis, showed a direct modulatory effect on late onset $\mathrm{AD}$ gene networks, which was verified not only in human brain but also in an experimental animal model. A salient finding was that the differential gene expression observed for late onset $\mathrm{AD}$ presented a distance-dependent relationship with TYROBP (Figure 1B). Those genes with a higher functional association with TYROBP are more likely to be altered during the disease process, as well as to propagate the pathologic effects to their connected neighbors. Although this characteristic gene network regulatory effect (Zhang et al., 2013) needs further exploration and validation in other neurodegenerative diseases, it illustrates how connectional links at the molecular level can mediate disease propagation.

\section{Inter-Cellular Misfolded Proteins Propagation Across Structural Pathways}

Proteins that fail to configure properly are called misfolded proteins (MP). Historically, they have been causally associated with aging and several human neurodegenerative diseases (Braak and Braak, 1991; Dobson, 2002, 2003; Braak et al., 2004). The prion-like hypothesis proposes that cell-to-cell transmission of toxic MP is a principal cause of neurodegeneration (Frost et al., 2009; Brundin et al., 2010; Hallbeck et al., 2013). Increasing neuropathologic evidence supports the spread of MPs from initial host regions to anatomically connected areas, spreading and simultaneously re-seeding the toxic effects (Frost et al., 2009; Waters, 2010; Nath et al., 2012; Jucker and Walker, 2013; Song et al., 2014). This fact, combined with recent evidence supporting the notion that each neurodegenerative disorder is associated with a characteristic group of MPs (Brundin et al., 2010), motivated in part the network degeneration hypothesis (NDH; Seeley et al., 2009). This hypothesis proposes that each disorder should present disease-specific anatomic, 
A

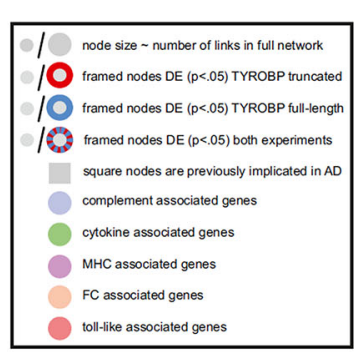

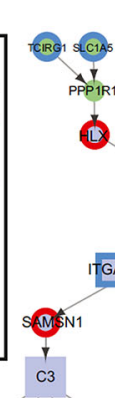

(1)

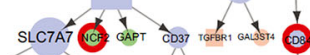

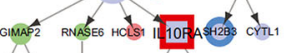

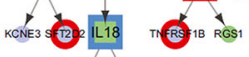
상 궁
FIGURE 1 | Connectivity distances to pathologic epicenters predict diseases effects. (A) TYROBP causal network in late onset Alzheimer's disease (AD), (B) differential expression levels of deregulated genes associated with TYROBP at various functional
B

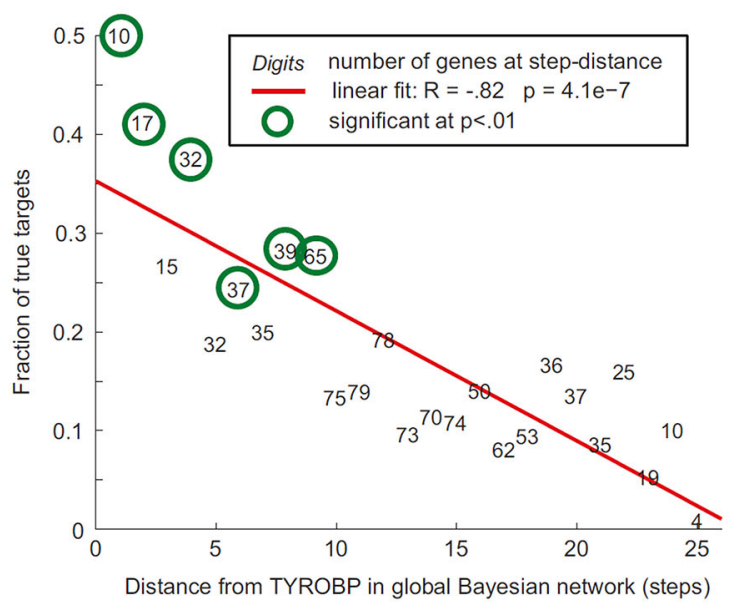

distances from it. Note the negative association $(R=-0.82$; $P<10^{-3}$ ) implying increasing impact with proximity to TYROBP. Figure adapted from Zhang et al. (2013), with permission from Elsevier.

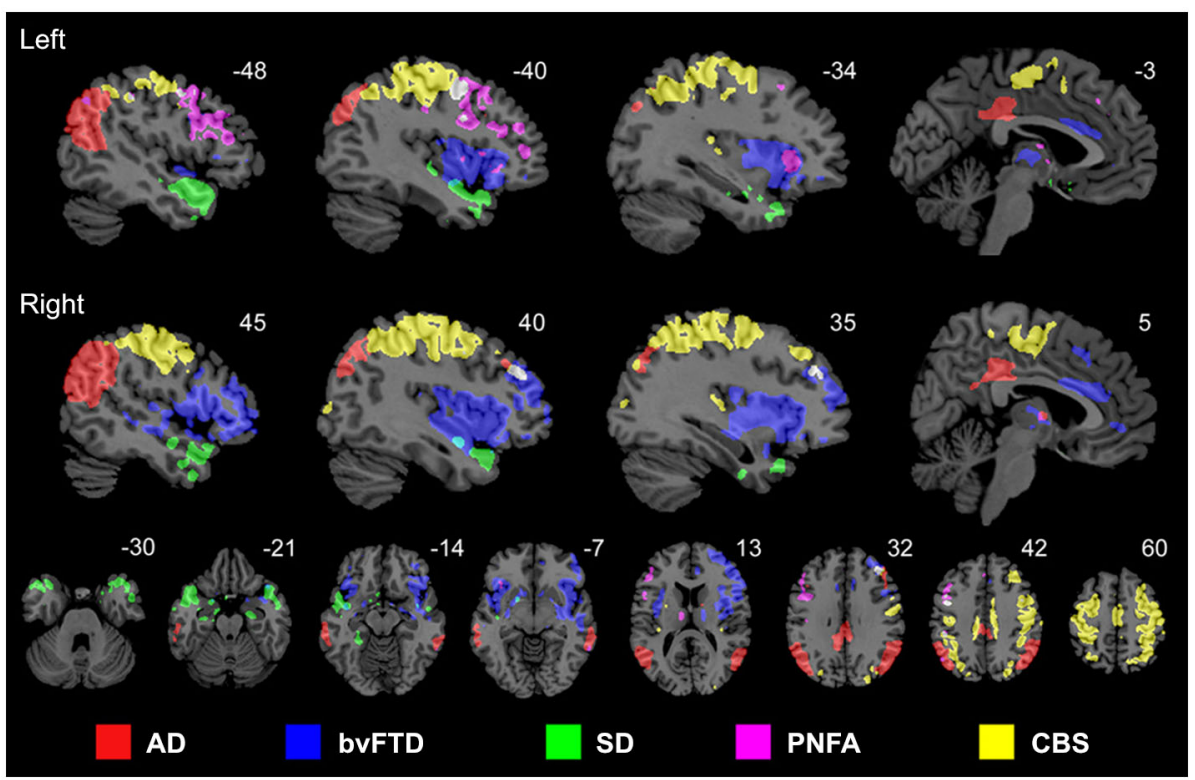

FIGURE 2 | Anatomically dissociable networks targeted by five different neurodegenerative disorders: $A D$, behavioral variant frontotemporal dementia (bvFTD), semantic dementia (SD), progressive nonfluent aphasia (PNFA), and corticobasal syndrome (CBS). Figure adapted from Seeley et al. (2009), with permission from Elsevier. functional and metabolic pathways. Seeley and colleagues used MRI to demonstrate that different neurodegenerative disorders are associated with spatially dissociable atrophy patterns, each pattern corresponding to a consistent structural covariance and functional sub-network (Figure 2; Seeley et al., 2009). In a complementary study (Zhou et al., 2012), the same group showed that regions with higher connectivity with, and shorter functional distances to, disease-specific epicenters presented greater structural atrophy. Raj et al., 2012, introduced a diffusion network model of intra-brain MP propagation, according to which the increase over time of the number of diseased afferents from a given brain region to any other region depends upon the disease concentration factor in both regions and upon the anatomical connection strength between them (Raj et al., 2012). From this model, an analytical expression for structural atrophy dynamics was obtained. After a mathematical 
decomposition of a healthy brain anatomical connectome, the authors found a significant correspondence between specific dissociable connectivity modules and the characteristic atrophy patterns of different neurodegenerative diseases ( $\mathrm{AD}$, behavioral variant FTD [bvFTD]). Each connectivity module's weight in the initial connectome was inversely proportional to the population prevalence of a specific disorder (AD, bvFTD or $\mathrm{HD})$. This suggested that the final structural atrophy pattern in adulthood could be the weighted combination of characteristic atrophy patterns from prevalent neurodegenerative diseases, in which each disease-characteristic pattern is weighted by the individual predisposition to express such disease. In general, these three seminal studies (Seeley et al., 2009; Raj et al., 2012; Zhou et al., 2012) supported the NDH, as well as the structural and functional connectivity-mediated spread of neuropathologic effects. However, neurodegenerative gray matter atrophy patterns may not be uniquely provoked by MP toxicity. Other pathologic factors, such as neuronal activity toxicity, and metabolic and vascular deregulations (see below) may contribute to cell death.

In order to obtain straight evidence of MP spread as a function of anatomical proximity to a disease propagation epicenter, Iturria-Medina et al. (2014), analyzed PET A $\beta$ deposition patterns in 733 healthy and diseased brains. Motivated by the remarkable similarity between intra-brain pathology propagation and the spread of human infectious diseases in social networks, we hypothesized that MP dynamics can be mathematically described and characterized by the epidemic-like interactions between infection agents (the aberrant proteins) and the brain's defense response, mediated by the brain's anatomical architecture (Iturria-Medina et al., 2014). The proposed epidemic spreading model (ESM) reproduced $A \beta$ patterns from healthy to advanced diseases states, allowing the reconstruction of individual lifetime histories of intra-brain $A \beta$ propagation, and the subsequent analysis of the biological factors that promote such propagation/deposition (e.g., the relationship of clinical state with MP production and/or clearance). When exploring the relation between regional $A \beta$ deposition pattern and the connectional proximity to the $A \beta$ outbreak regions, as identified by the ESM (anterior and posterior cingulate cortices), a significant negative linear trend was observed (Figure 3A), with more advanced disease states corresponding to higher deposition. Also, a significant negative relation between regional anatomical connectivity degree and $\mathrm{A} \beta$ arrival time (measures

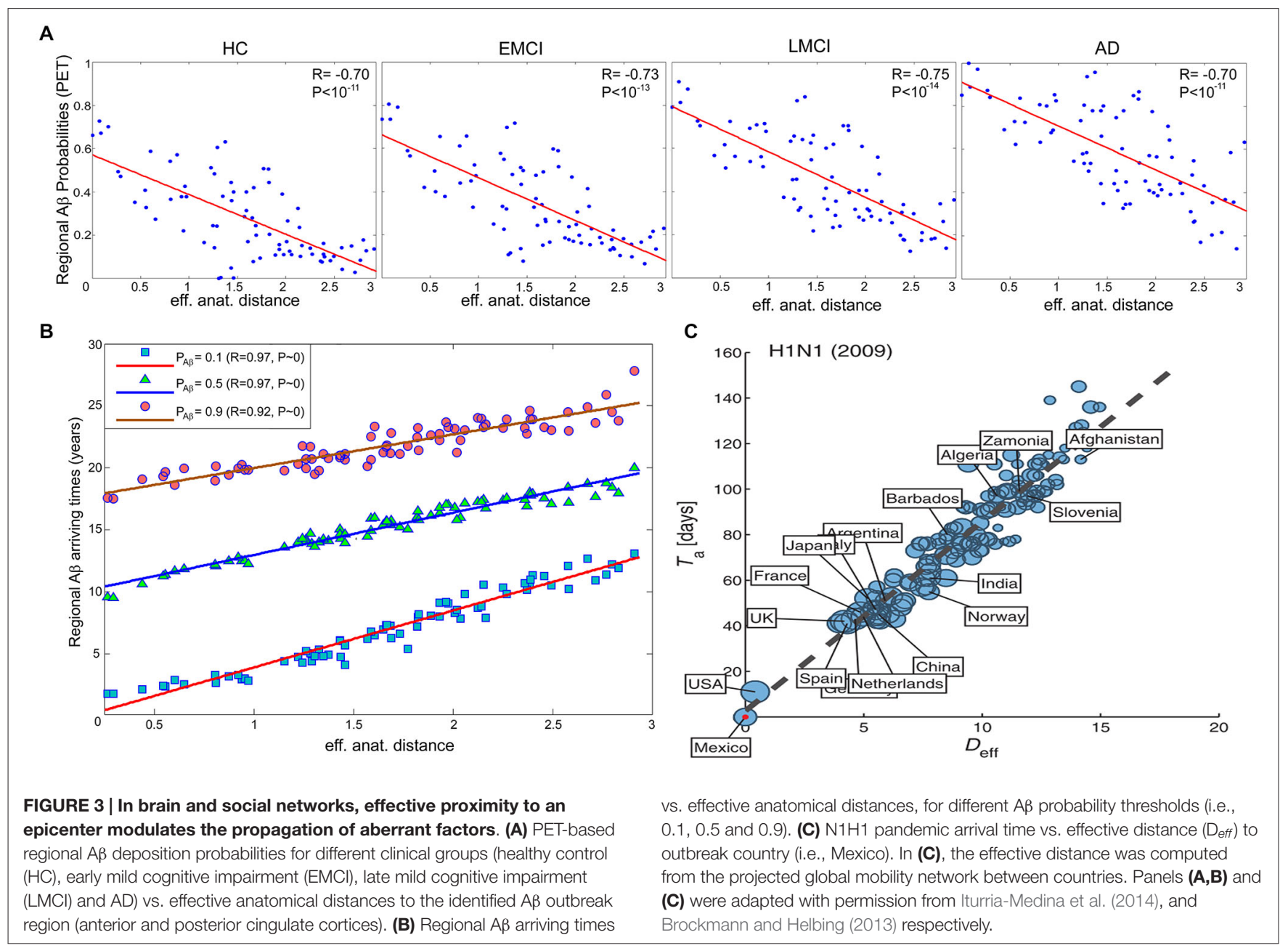


of hubness and disease vulnerability) was observed (Figure 3B). This relation was independent of the selection of different $A \beta$ deposition thresholds, indicating that regions with a higher degree of anatomical connectivity experience earlier $A \beta$ arrival and, consequently, larger periods of exposure to the toxic effect of the aberrant protein. Interestingly, and supporting the hypothesis of an epidemic spreading behavior for MP propagation, a similar linear predictive relationship has been reported for effective distance in human social networks and disease arrival times for real epidemic propagation of infectious disease (Brockmann and Helbing, 2013) (e.g., 2009 H1N1 pandemic; see Figure 3C).

Also in line with the prion-like hypothesis, the phosphorylated $43 \mathrm{kDa}$ TAR DNA-binding protein (pTDP43) has been identified as a major neuropathologic factor in ALS and frontotemporal lobar degeneration (Neumann et al., 2006; Geser et al., 2009). Recently, Brettschneider et al. (2013), identified four characteristic stages of pTDP-43 neuropathology in ALS, which suggested a sequential pTDP-43 intra-brain dissemination pattern (Brettschneider et al., 2013). Schmidt et al. (2015), found a dense level of anatomical connectivity between the regions of these four pTDP-43 stages. These authors also used a computational random walker spread model to simulate axonal spread of the pTDP-43 factor as a walking particle along the white matter pathways (Schmidt et al., 2015). Consistent with the hypothesis that pTDP-43 pathology is propagated along axonal pathways, they observed a significant overlap between the simulated pTDP-43 patterns and the sequential distribution found previously in ALS autopsy cases.

\section{Neuronal Activity-Dependent Neurodegeneration}

The upstream causal role of MP on neurodegenerative disorders is currently under scientific controversy (Soto and Castilla, 2004; Hilker et al., 2011). MP presence do not always correlate well with structural atrophy and/or cognitive decline levels, whereas therapeutic drugs created to reduce MP levels have demonstrated poor modulatory effects on disease progression (Holmes et al., 2008). Different alternative hypotheses have been proposed in order to fit the inconsistencies of the MP prionlike assumptions. For example, the Caspase- 6 neurodegeneration hypothesis of AD (LeBlanc et al., 1999; Albrecht et al., 2007; LeBlanc, 2013), explains cell inflammation and death by the stress-associated action of the Caspase- 6 enzyme. Caspase- 6 activation modulates also $A \beta$ and phosphorylated tau concentrations, which are strongly associated to the stress in neurons and cell lines. Moreover, consistent evidence suggests that abnormal neuronal and synaptic activity may modulate brain MP levels (Kamenetz et al., 2003; Cirrito et al., 2005, 2008; Buckner et al., 2009; Bero et al., 2011). For instance, exogenous increases in neuronal and synaptic activity in the hippocampus, induced by electric stimulation, increase the extracellular $\mathrm{A} \beta$ concentrations in that region (Cirrito et al., 2005). Also, endogenous neuronal activity changes have an equivalent impact on $\mathrm{A} \beta$ concentrations (Bero et al., 2011), suggesting that regional differences in basal neuronal activity levels could explain regional vulnerabilities to $A \beta$ presence and toxicity. From these facts arise some relevant questions: can aberrant neuronal/synaptic activity have an upstream role in neurodegenerative progression, and, importantly, is functional connectivity a mediator of neuronal/synaptic toxicity spreading? Motivated by these questions, de Haan et al. (2012), used neural mass modeling to explore local neuronal activity in relation to large-scale connectivity in normal and abnormal conditions. For this, the authors simulated neural dynamics using a real structural brain connectome, and induced progressive damage to the regions based on their level of activity. The results suggested that, in no-task conditions, hubs should be the most active regions (due to the convergence of heteromodal activity), and also that excessive connectivitydependent neuronal activity can have a significant role in the neurodegenerative progression, thus explaining the associated hub vulnerability (de Haan et al., 2012). Previously, a robust relationship between regional hubness (in terms of functional connectivity) and $A \beta$ depositions had been reported (Buckner et al., 2009), whereas functional hyperconnectivity, mainly between cingulate and medio-temporal regions, had been associated with semantic memory deficits (Gardini et al., 2015). Similarly, in $\mathrm{AD}$, regional metabolic alterations had been found to follow $\mathrm{A} \beta$ presence in many brain regions (Förster et al., 2012), whereas the spatial mismatch between these two pathologic components can be explained by functional connection to $\mathrm{A} \beta$ binding areas (Klupp et al., 2014). This means that non- $\mathrm{A} \beta$ areas can also be metabolically deregulated during disease progression if those areas are functionally linked to $A \beta$ and/or functionally-impaired zones. Moreover, derangement of metabolic connectivity patterns have been associated with elevated $\mathrm{A} \beta$ burden (Carbonell et al., 2014a,b), while a significant modulatory impact of the Apoes 4 genotype on hypometabolism had been observed (Jagust and Landau, 2012; Carbonell et al., 2014a). All together, these results support the contention that neuronal/synaptic toxicity spreading in neurodegeneration, and associated activity-dependent deregulation of local MP and metabolic levels, strongly depend on anatomic, functional and metabolic brain connectional patterns.

\section{Towards a Multi-Factorial Disease Spreading Perspective}

Although generally associated with specific hypotheses, previously proposed pathologic mechanisms are not unrelated. In addition to have a modulatory impact on protein expression, gene activity is markedly associated with structural connectivity patterns (French and Pavlidis, 2011; Wolf et al., 2011a; Ji et al., 2014; Fakhry and Ji, 2015) and synaptic density dynamics (Goyal and Raichle, 2013). This suggests that alterations in gene regulatory networks or aberrant signal spreading across them may induce important changes in structural, functional and metabolic brain patterns, even as an additional downstream effect of a main genetic pathologic factor. Similarly, strong associations persist among different forms of brain connectivity, under normal or abnormal conditions. The vascular and metabolic/functional systems represent a remarkable example. Among other relevant functions, the vascular system supplies oxygen, glucose and other nutrients, and clears away deoxygenated blood and metabolic products (Scremin, 2012). These functions are essential to satisfy daily 

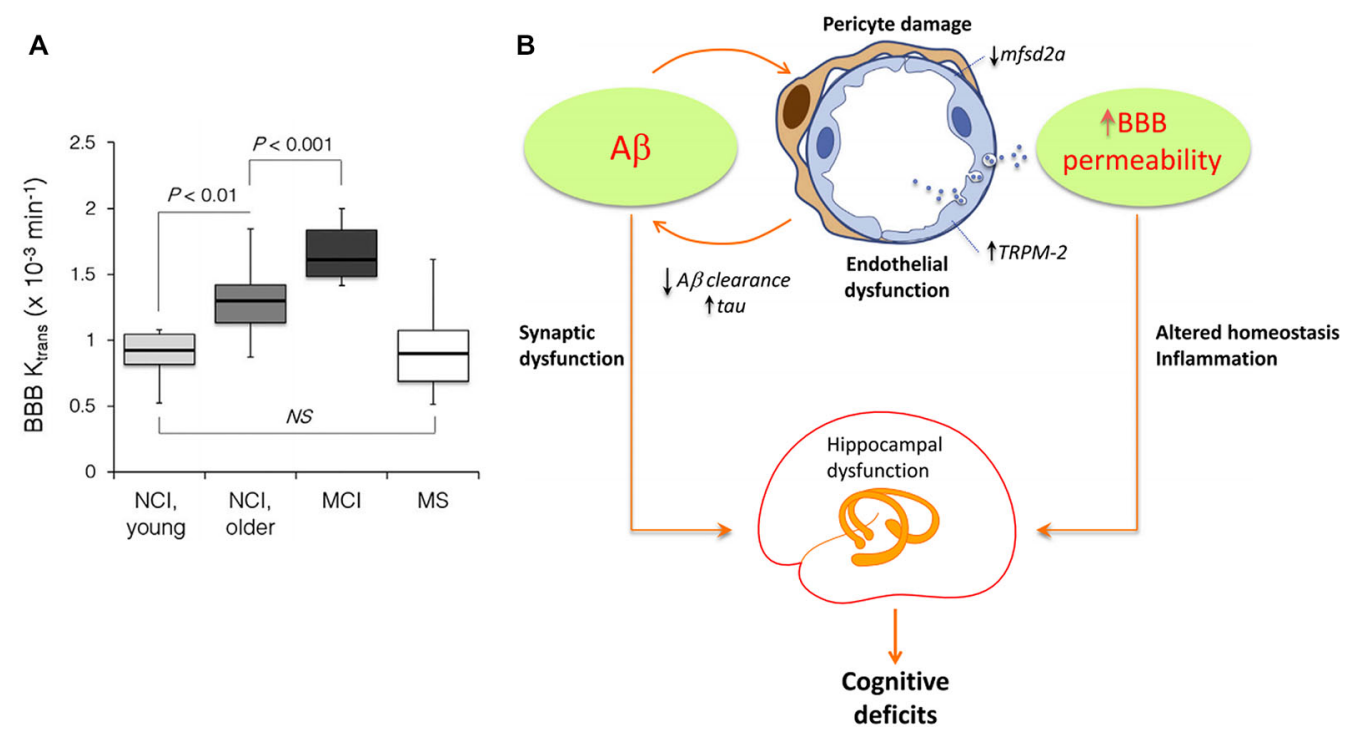

FIGURE 4 | Age-dependent blood-brain barrier (BBB) permeability breakdown may be causally associated with neurodegenerative spreading (Panels (A) and (B) were adapted from Montagne et al. (2015), ladecola (2015), respectively, with permission from Elsevier) (A) Significant increases in BBB permeability in older compared young group of individuals with no cognitive impairment $(\mathrm{NCl})$, and $\mathrm{MCl}$ compared to older $\mathrm{NCl}$ group in the entire hippocampus. Multiple sclerosis (MS) patients with no cognitive impairment were comparable with the age-matched young $\mathrm{NCl}$ group (Montagne et al., 2015). (B) Hypothetical pathologic mechanisms by which A $\beta$ may induce BBB permeability alterations and hippocampal/cognitive dysfunction (ladecola, 2015). A $\beta$ affects endothelial cells, damaging pericytes, vesicular transport and $\mathrm{Ca}^{+}$balance. This contributes to BBB disruption, homeostasis alterations, reduction on misfolded proteins (MP) clearance and tentatively to hippocampal dysfunction, cognitive deficits and intra-brain pathology spreading. neuronal/glial energy and maintenance demands. However, this close association dates from initial neurodevelopmental processes: axon-guidance cues mediate the navigation of blood vessels along predestined tracks during development (Carmeliet and Tessier-Lavigne, 2005; Zacchigna et al., 2008), whereas angiogenic vascular endothelial growth factor regulates the migration of various neuron types to their final destination (Schwarz et al., 2004; Zacchigna et al., 2008). Recently, Lacoste et al. (2014), combined genetics, imaging and computational tools to verify that neural activity changes can modulate vascular networks. They found that decrease or enhancement of neural activity (by deafferentation, (de)stimulation or genetic impairment of neurotransmitter release) leads to equivalent effects in vascular density and branching (Lacoste et al., 2014). Together, these facts explain the anatomical positioning and behavioral similarities that have also been uncovered among the vascular and the functional/metabolic pathways (Melie-García et al., 2013; Jann et al., 2015). Moreover, the vascular system plays a major role in aging and associated neurodegenerative processes (Zacchigna et al., 2008; Quaegebeur et al., 2011; Iadecola, 2013). Capillary density loss and other vascular abnormalities have been consistently observed in healthy aging, $\mathrm{AD}$, leukoaraiosis (LA) and HD (Brown and Thore, 2011; Wolf et al., 2011b). Damage to vascular network integrity leads to MP clearance deficits and resultant deposition. For instance, the efflux across the blood-brain barrier (BBB) contributes $A \beta$ clearance (Deane et al., 2009; Qosa et al., 2014). Qosa et al. (2014), reported that around a $60 \%$ of soluble $A \beta^{40}$ is cleared across $\mathrm{BBB}$ while the remaining is cleared by brain degradation. Consistent with this thesis, a significant age-dependent BBB permeability breakdown, that correlates with cognitive dysfunction, has been observed in human hippocampus (Montagne et al., 2015; see Figure 4A). Such aging effects have a crucial impact on BBB-mediated MP clearance and deposition (Iadecola, 2015; see Figure 4B), contributing to structural, functional and metabolic connectional deregulation in a continuous degenerative cycle. In addition, brain neuroinflammation is characteristic feature during neurodegeneration (Streit et al., 2004; Block et al., 2007; Lull and Block, 2010). It is particularly associated to microglia cells activity, which reacts defensively in respond to different events, such as infection, brain injury or associated autoimmune processes (Gendelman, 2002). Under certain pathologic conditions (ex. presence of environmental toxins or neuronal damage), microglias can enter to a hyperactivation state and release excessive reactive oxygen species (ROS), which cause neurotoxicity and cell death (Block et al., 2007; Lull and Block, 2010). Then, local pathologic effects associated to microglia-mediated neuroinflammatory processes may impact other connected areas. Similarly that with the region-region transmission of previously mentioned aberrant factors (ex. MP, toxic neuronal/synaptic signals, metabolic deregulation, BBB damage), functional/metabolic impairment and neuronal death in a given brain region, due to neuroinflammation and ROS, may alter its vascular, functional, metabolic and anatomical links, and gradually the multi-factorial subnetworks associated to the connected regions, extending the negative neuroinflammatory effects across the interconnected brain. 


\section{Discussion and Conclusions}

Converging evidence supports the central role of brain connectivity in neurodegenerative progression. Abnormal connectivity might not only be involved in the propagation of downstream effects, it might also support upstream pathologic causes (Pievani et al., 2014). This supports the strategic importance of understanding the role of brain connectivity in disease evolution. Notably, the finding of patterns of pathology that reflect known structural connectivity, suggests the active role of specific epicenter nodes during the disease processes (e.g., deregulated genes, cell assemblies, and/or gross regions). In social networks, the presence of individuals with a disproportionately large number of contacts (social hubs) accelerates considerably the spread of infectious disease (Newman, 2002; Lloyd-Smith et al., 2005; Leventhal et al., 2015). This hub-centric behavior could be also be a feature of intra-brain pathologic propagations, not only limited to neurodegeneration but also present in other disorders (e.g., schizophrenia, epilepsy, Asperger's syndrome). A recent meta-analysis study of 26 different brain diseases showed that disease-specific structural lesions were mainly located on

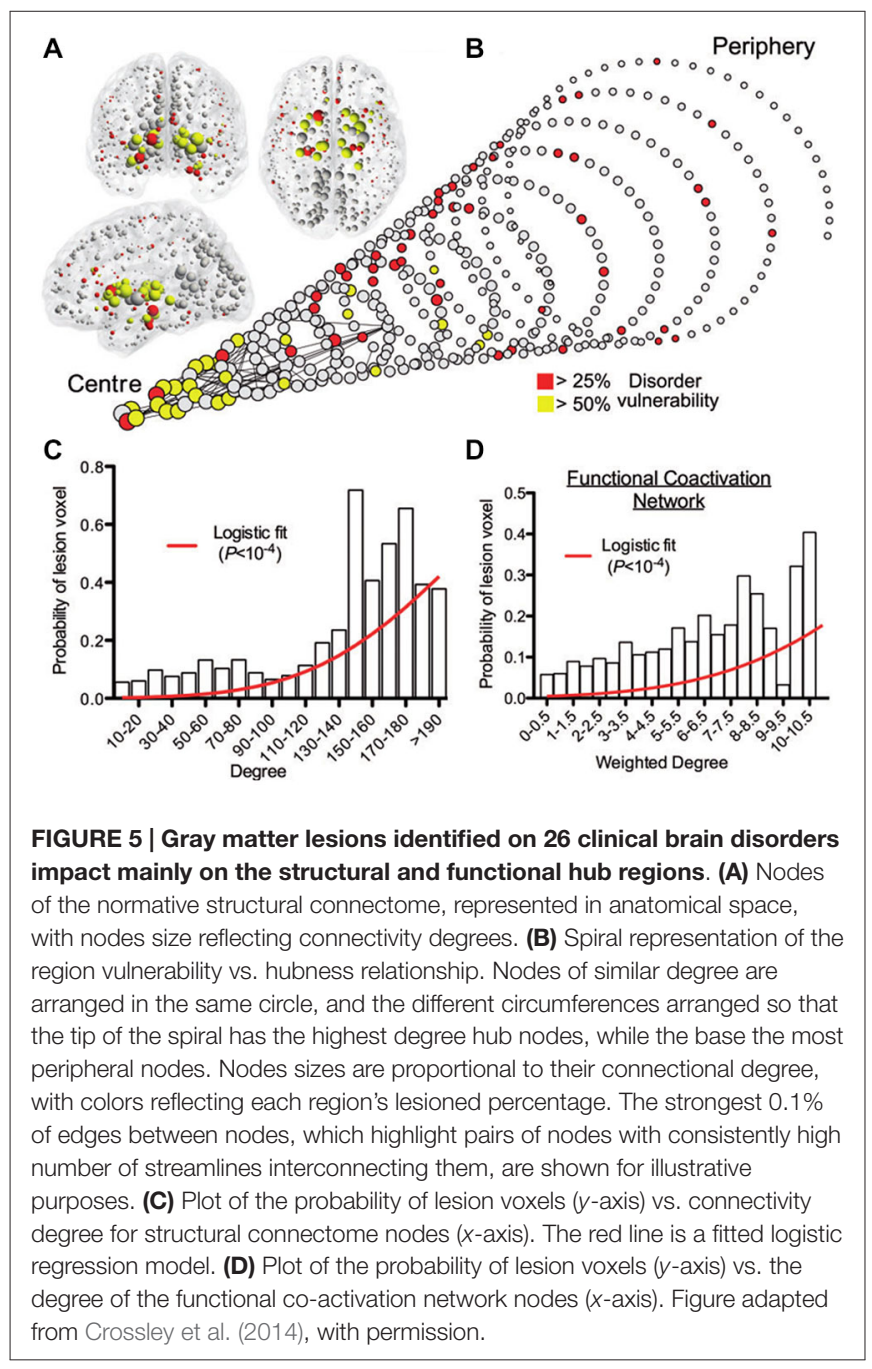

connectivity hub regions (see Figure 5). This hub vulnerability could be a consequence of the high topological centrality and biological cost of the hubs, that make them more sensitive to a diverse range of pathogenic processes (Crossley et al., 2014). In addition, we showed that brain regions with a higher degree of anatomical connectivity experience early $A \beta$ arrival and larger periods of $A \beta$ exposition (Figure 3B; Iturria-Medina et al., 2014), which, in addition to excessive connectivity-dependent neuronal activity (de Haan et al., 2012), explains the higher $\mathrm{A} \beta$ deposition levels found on functional hubs (Buckner et al., 2009).

In spite of its biological relevance, it is not totally clear yet how to quantify the role of dynamic connectivity in disease evolution. For instance, although MP propagation have been modeled and studied by means of diffusion networks (Raj et al., 2012) and epidemic-like spreading (Iturria-Medina et al., 2014), the predictive power of these models still needs further validation. Similarly, there are promising advances in the modeling and understanding of neuronal/synaptic spreading across structural networks (Sotero et al., 2007; Valdes-Sosa et al., 2009; Sanz-Leon et al., 2013, 2015; Messé et al., 2015), but these methodologies also require additional predictive validation. Increasing evidence supports that gender have a substantial impact on structural and functional brain connectivity (Gong et al., 2011), which have been suggested to explain specific gender-related cognitive differences (Ingalhalikar et al., 2014). Gender is also associated to the risk of develop specific neurodegenerative diseases. For example, women are more likely to develop AD than men (Farrer et al., 1997; Damoiseaux et al., 2012), whereas men present a significant higher risk to develop PD (Wooten et al., 2004). Thus, in order to reach a realistic operational model of dynamic brain organization during aging and degeneration, it is essential to clarify how gender-related differences, from genetic, molecular, structural and/or functional levels, might modulate the role of brain connectivity in disease development and progression. I addition, it is still unclear if a given connectivity change should be interpreted as the result of a pathologic induced alteration or as the outcome of a compensatory change. The current lack of multi-factorial trajectory analysis and particularly the absence of robust causal models of disease progression, make still unfeasible to discriminate between connectivity associated upstream and downstream effects. A decreased connection could be reflecting either a pathologic alteration or a counteracting compensation mechanism, whereas an increased connection could be responding either to a compensatory mechanism or to a pathologic spreading effect. Importantly, it is also unclear how our understanding of the role of connectivity in disease progression could be translated into the development of effective therapeutic strategies. As Zhang et al. (2013) point out, targeting highly-connected genes in deregulated gene networks may be effective in disrupting disease-related networks for the purpose of therapy, but that could be at the cost of unknown adverse effects. How to diminish the outcome of negative effects after possible therapeutic interventions is still the subject of much scientific debate, Computer simulation modeling could help considerably the exploration of the effects of intervention strategies. For example, Proctor et al. (2013) modeled DNA 
damage, p53/GSK3 regulation, $A \beta$ and tau dynamics to predict the intervention effects of $A \beta$ immunization. However, in order to extend the simulation analyses, we will need a deeper understanding of the genetic, protean, metabolic, vascular, functional and structural aberrant interactions associated with aging and neurodegeneration.

\section{References}

Albrecht, S., Bourdeau, M., Bennett, D., Mufson, E. J., Bhattacharjee, M., and LeBlanc, A. C. (2007). Activation of caspase- 6 in aging and mild cognitive impairment. Am. J. Pathol. 170, 1200-1209. doi: 10.2353/ajpath.2007. 060974

Al-Chalabi, A., and Hardiman, O. (2013). The epidemiology of ALS: a conspiracy of genes, environment and time. Nat. Rev. Neurol. 9, 617-628. doi: 10. 1038/nrneurol.2013.203

Axer, M., Amunts, K., Grässel, D., Palm, C., Dammers, J., Axer, H., et al. (2011). A novel approach to the human connectome: ultra-high resolution mapping of fiber tracts in the brain. Neuroimage 54, 1091-1101. doi: 10.1016/j.neuroimage. 2010.08.075

Bernot, G., Comet, J., Richard, A., Chaves, M., and Gouz, J. (2013). "Modeling in computational biology and biomedicine," in A Multidisciplinary Endeavor, eds F. Cazals and P. Kornprobst (Berlin, Heidelberg: Springer Berlin Heidelberg), 47-80.

Bero, A. W., Yan, P., Roh, J. H., Cirrito, J. R., Stewart, F. R., Raichle, M. E., et al. (2011). Neuronal activity regulates the regional vulnerability to amyloid- b deposition. Nat. Neurosci. 14, 750-756. doi: 10.1038/nn.2801

Bicchi, I., Morena, F., Montesano, S., Polidoro, M., and Martino, S. (2013). MicroRNAs and molecular mechanisms of neurodegeneration. Genes (Basel) 4, 244-263. doi: 10.3390/genes4020244

Block, M. L., Zecca, L., and Hong, J.-S. (2007). Microglia-mediated neurotoxicity: uncovering the molecular mechanisms. Nat. Rev. Neurosci. 8, 57-69. doi: 10. 1038/nrn2038

Bota, M., Dong, H., and Swanson, L. W. (2003). From gene networks to brain networks. Nat. Neurosci. 6, 795-799. doi: 10.1038/nn1096

Bouchard-Mercier, A., Paradis, A.-M., Rudkowska, I., Lemieux, S., Couture, P., and Vohl, M.-C. (2013). Associations between dietary patterns and gene expression profiles of healthy men and women: a cross-sectional study. Nutr. J. 12:24. doi: 10.1186/1475-2891-12-24

Braak, H. B. E., and Braak, E. (1991). Neuropathological stageing of Alzheimer-related changes. Acta Neuropathol. 82, 239-259. doi: 10.1007/bf003 08809

Braak, H., Ghebremedhin, E., Rüb, U., Bratzke, H., and Del Tredici, K. (2004). Stages in the development of Parkinson's disease-related pathology. Cell Tissue Res. 318, 121-134. doi: 10.1007/s00441-004-0956-9

Brettschneider, J., Del Tredici, K., Toledo, J. B., Robinson, J. L., Irwin, D. J., Grossman, M., et al. (2013). Stages of pTDP-43 pathology in amyotrophic lateral sclerosis. Ann. Neurol. 74, 20-38. doi: 10.1002/ana.23937

Brockmann, D., and Helbing, D. (2013). The hidden geometry of complex, network-driven contagion phenomena. Science 342, 1337-1342. doi: 10 . 1126/science. 1245200

Brown, W., and Thore, C. (2011). Cerebral microvascular pathology in aging and neurodegeneration. Neuropathol. Appl. Neurobiol. 37, 56-74. doi: 10.1111/j. 1365-2990.2010.01139.x

Brundin, P., Melki, R., and Kopito, R. (2010). Prion-like transmission of protein aggregates in neurodegenerative diseases. Nat. Rev. 11, 301-307. doi: 10. 1038/nrm 2873

Buckholtz, J. W., and Meyer-Lindenberg, A. (2012). Psychopathology and the human connectome: toward a transdiagnostic model of risk for mental illness. Neuron 74, 990-1004. doi: 10.1016/j.neuron.2012.06.002

Buckner, R. L., Sepulcre, J., Talukdar, T., Krienen, F. M., Liu, H., Hedden, T., et al. (2009). Cortical hubs revealed by intrinsic functional connectivity: mapping, assessment of stability and relation to Alzheimer's disease. J. Neurosci. 29, 1860-1873. doi: 10.1523/JNEUROSCI.5062-08. 2009

\section{Acknowledgments}

We are grateful to the anonymous referees for their helpful comments. This work was partially supported by Brain Canada and the Azrieli Foundation, grant number BC_Azrieli_MIRI_3388.

Carbonell, F., Charil, A., Zijdenbos, A. P., Evans, A. C., and Bedell, B. J. (2014a) $\beta$-Amyloid is associated with aberrant metabolic connectivity in subjects with mild cognitive impairment. J. Cereb. Blood Flow Metab. 34, 1169-1179. doi: 10. 1038/jcbfm.2014.66

Carbonell, F., Charil, A., Zijdenbos, A. P., Evans, A. C., Bedell, B. J., and Alzheimer's Disease Neuroimaging Initiative. (2014b). Hierarchical multivariate covariance analysis of metabolic connectivity. J. Cereb. Blood Flow Metab. 34, 1936-1943. doi: 10.1038/jcbfm.2014.165

Carmeliet, P., and Tessier-Lavigne, M. (2005). Common mechanisms of nerve and blood vessel wiring. Nature 436, 193-200. doi: 10.1038/nature03875

Cirrito, J. R., Kang, J., Lee, J., Stewart, F. R., Verges, D. K., Silverio, L. M., et al. (2008). Endocytosis is required for synaptic activity-dependent release of amyloid- $\beta$ in vivo. Neuron 58, 42-51. doi: 10.1016/j.neuron.2008.02.003

Cirrito, J. R., Yamada, K. A., Finn, M. B., Sloviter, R. S., Bales, K. R., May, P. C., et al. (2005). Synaptic activity regulates interstitial fluid amyloid- $\beta$ levels in Vivo. Neuron 48, 913-922. doi: 10.1016/j.neuron.2005.10.028

Colman, R. J., Anderson, R. M., Johnson, S. C., Kastman, E. K., Simmons, H. A., Kemnitz, J. W., et al. (2009). Caloric restriction delays disease onset and mortality in rhesus monkeys. Science 25, 201-204. doi: 10.1126/science. 1173635

Cramer, P., Cirrito, J., Wesson, D., Lee, C., Karlo, J., Zinn, A., et al. (2012). ApoEdirected therapeutics rapidly clear $\beta$-amyloid and reverse deficits in AD mouse models. Science 335, 1503-1506. doi: 10.1126/science.1217697

Crespo, I., Roomp, K., Jurkowski, W., Kitano, H., and del Sol, A. (2012). Gene regulatory network analysis supports inflammation as a key neurodegeneration process in prion disease. BMC Syst. Biol. 6:132. doi: 10.1186/1752-0509-6-132

Crichton, G. E., Bryan, J., and Murphy, K. J. (2013). Dietary antioxidants, cognitive function and dementia-a systematic review. Plant Foods Hum. Nutr. 68, 279-292. doi: 10.1007/s11130-013-0370-0

Crossley, N. A., Mechelli, A., Scott, J., Carletti, F., Fox, P. T., McGuire, P., et al. (2014). The hubs of the human connectome are generally implicated in the anatomy of brain disorders. Brain 137, 2382-2395. doi: 10.1093/brain/ awu132

Damoiseaux, J., Seeley, W., Zhou, J., Shirer, W., Coppola, G., Karydas, A., et al. (2012). Gender modulates the APOE $\varepsilon 4$ effect in healthy older adults: convergent evidence from functional brain connectivity and spinal fluid tau levels. J. Neurosci. 32, 8254-8262. doi: 10.1523/JNEUROSCI.0305-12.2012

Deane, R., Bell, R., Sagare, A., and Zlokovic, B. (2009). Clearance of amyloidbeta Alzheimer's, peptide across the blood-brain barrier: implication for therapies in disease. CNS Neurol. Disord. Drug Targets 8, 16-30. doi: 10. 2174/187152709787601867

de Haan, W., Mott, K., van Straaten, E. C. W., Scheltens, P., and Stam, C. J. (2012). Activity dependent degeneration explains hub vulnerability in Alzheimer's disease. PLoS Comput. Biol. 8:e1002582. doi: 10.1371/journal.pcbi.1002582

Dobson, C. M. (2002). Protein misfolding diseases: getting out of shape. Nature 418, 729-730. doi: 10.1038/418729a

Dobson, C. M. (2003). Protein folding and misfolding. Nature 426, 884-890. doi: $10.1038 /$ nature 02261

Evans, A. C. (2013). Networks of anatomical covariance. Neuroimage 80, 489-504. doi: 10.1016/j.neuroimage.2013.05.054

Fakhry, A., and Ji, S. (2015). High-resolution prediction of mouse brain connectivity using gene expression patterns. Methods 73C, 71-78. doi: 10 . 1016/j.ymeth.2014.07.011

Farrer, L., Cupples, L., Haines, J., Hyman, B., Kukull, W., Mayeux, R., et al. (1997). Effects of age, sex and ethnicity on the association between apolipoprotein $\mathrm{E}$ genotype and Alzheimer disease: a meta-analysis. APOE and Alzheimer disease meta analysis consortium. JAMA 278, 1349-1356. doi: 10.1001/jama.278. 16.1349 
Förster, S., Grimmer, T., Miederer, I., Henriksen, G., Yousefi, B. H., Graner, P., et al. (2012). Regional expansion of hypometabolism in Alzheimer's disease follows amyloid deposition with temporal delay. Biol. Psychiatry 71, 792-797. doi: 10.1016/j.biopsych.2011.04.023

French, L., and Pavlidis, P. (2011). Relationships between gene expression and brain wiring in the adult rodent brain. PLoS Comput. Biol. 7:e1001049. doi: 10. 1371/journal.pcbi.1001049

Friston, K. J. (2011). Functional and effective connectivity: a review. Brain Connect. 1, 13-36. doi: 10.1089/brain.2011.0008

Frost, B., Jacks, R. L., and Diamond, M. I. (2009). Propagation of tau misfolding from the outside to the inside of a cell. J. Biol. Chem. 284, 12845-12852. doi: 10. 1074/jbc.m808759200

Gallou-Kabani, C., Vigé, A., Gross, M. S., and Junien, C. (2007). Nutriepigenomics: lifelong remodelling of our epigenomes by nutritional and metabolic factors and beyond. Clin. Chem. Lab. Med. 45, 321-327. doi: 10. 1515/cclm.2007.081

Gardini, S., Venneri, A., Sambataro, F., Cuetos, F., Fasano, F., Marchi, M., et al. (2015). Increased functional connectivity in default mode network in mild cognitive impairment: a maladaptive compensatory mechanism associated with poor semantic memory performance. J. Alzheimers Dis. 45, 457-470. doi: 10. 3233/JAD-142547

Gendelman, H. E. (2002). Neural immunity: friend or foe? J. Neurovirol. 8, 474-479. doi: 10.1080/13550280290168631

Genin, E., Hannequin, D., Wallon, D., Sleegers, K., Hiltunen, M., Combarros, O., et al. (2011). APOE and Alzheimer disease: a major gene with semi-dominant inheritance. Mol. Psychiatry 16, 903-907. doi: 10.1038/mp.2011.52

Geser, F., Martinez-lage, M., Kwong, L. K., Lee, V. M., and Trojanowski, J. Q. (2009). Amyotrophic lateral sclerosis, frontotemporal dementia and beyond: the TDP-43 diseases. J. Neurol. 256, 1205-1214. doi: 10.1007/s00415-0095069-7

Gomez-Ramirez, J., and Wu, J. (2014). Network-based biomarkers in Alzheimer's disease: review and future directions. Front. Aging Neurosci. 6:12. doi: 10. 3389/fnagi.2014.00012

Gong, G., He, Y., and Evans, A. C. (2011). Brain connectivity: gender makes a difference. Neuroscientist 17, 575-591. doi: 10.1177/10738584103 86492

Goyal, M. S., and Raichle, M. E. (2013). Gene expression-based modeling of human cortical synaptic density. Proc. Natl. Acad. Sci. U S A 110, 6571-6576. doi: 10. 1073/pnas.1303453110

Hagmann, P., Kurant, M., Gigandet, X., Thiran, P., Wedeen, V. J., Meuli, R., et al. (2007). Mapping human whole-brain structural networks with diffusion MRI. PLoS One 2:e597. doi: 10.1371/journal.pone.0000597

Hallbeck, M., Nath, S., and Marcusson, J. (2013). Neuron-to-neuron transmission of neurodegenerative pathology. Neuroscientist 19, 560-566. doi: 10. $1177 / 1073858413494270$

He, Y., and Evans, A. (2014). Magnetic resonance imaging of healthy and diseased brain networks. Front. Hum. Neurosci. 8:890. doi: 10.3389/fnhum.2014.00890

Hilker, R., Brotchie, J. M., and Chapman, J. (2011). Pros and cons of a prion-like pathogenesis in Parkinson's disease. BMC Neurol. 11:74. doi: 10.1186/14712377-11-74

Holmes, C., Boche, D., Wilkinson, D., Yadegarfar, G., Hopkins, V. B., Bayer, A., et al. (2008). Long-term effects of Abeta42 immunisation in Alzheimer's disease: follow-up of a randomised, placebo-controlled phase I trial. Lancet 372, 216-223. doi: 10.1016/S0140-6736(08)61075-2

Iadecola, C. (2013). The pathobiology of vascular dementia. Neuron 80, 844-866. doi: 10.1016/j.neuron.2013.10.008

Iadecola, C. (2015). Dangerous leaks: blood-brain barrier woes in the aging hippocampus. Neuron 85, 231-233. doi: 10.1016/j.neuron.2014.12.056

Ingalhalikar, M., Smith, A., Parker, D., Satterthwaite, T. D., Elliott, M. A., Ruparel, K., et al. (2014). Sex differences in the structural connectome of the human brain. Proc. Natl. Acad. Sci. U S A 111, 823-828. doi: 10.1073/pnas. 1316909110

Iturria-Medina, Y. (2013). Anatomical brain networks on the prediction of abnormal brain states. Brain Connect. 3, 1-21. doi: 10.1089/brain.2012. 0122

Iturria-Medina, Y., Canales-Rodríguez, E. J., Melie-García, L., Valdés-Hernández, P. A., Martínez-Montes, E., Alemán-Gómez, Y., et al. (2007). Characterizing brain anatomical connections using diffusion weighted MRI and graph theory. Neuroimage 36, 645-660. doi: 10.1016/j.neuroimage.2007.02.012
Iturria-Medina, Y., Sotero, R. C., Toussaint, P. J., Evans, A. C., and ADNI (2014). Epidemic spreading model to characterize misfolded proteins propagation in aging and associated neurodegenerative disorders. PLoS Comput. Biol. 10:e1003956. doi: 10.1371/journal.pcbi.1003956

Jagust, W. J., Landau, S. M., and Alzheimer's Disease Neuroimaging Initiative. (2012). Apolipoprotein E, not fibrillar $\beta$-amyloid, reduces cerebral glucose metabolism in normal aging. J. Neurosci. 32, 18227-18233. doi: 10 . 1523/JNEUROSCI.3266-12.2012

Jann, K., Gee, D., Kilroy, E., Schwab, S., Smith, R., Cannon, T., et al. (2015). Functional connectivity in BOLD and CBF data: similarity and reliability of resting brain networks. Neuroimage 106, 111-122. doi: 10.1016/j.neuroimage. 2014.11.028

Ji, S., Fakhry, A., and Deng, H. (2014). Integrative analysis of the connectivity and gene expression atlases in the mouse brain. Neuroimage 84, 245-253. doi: 10 . 1016/j.neuroimage.2013.08.049

Joseph, J., Cole, G., Head, E., and Ingram, D. (2009). Nutrition, brain aging and neurodegeneration. J. Neurosci. 29, 12795-12801. doi: 10.1523/JNEUROSCI. 3520-09.2009

Jucker, M., and Walker, L. C. (2013). Self-propagation of pathogenic protein aggregates in neurodegenerative diseases. Nature 501, 45-51. doi: 10. 1038/nature12481

Kamenetz, F., Tomita, T., Hsieh, H., Seabrook, G., Borchelt, D., Iwatsubo, T., et al. (2003). APP processing and synaptic function. Neuron 37, 925-937. doi: 10 1016/s0896-6273(03)00124-7

Karlebach, G., and Shamir, R. (2008). Modelling and analysis of gene regulatory networks. Nat. Rev. Mol. Cell Biol. 9, 770-780. doi: 10.1038/nrm2503

Klupp, E., Förster, S., Grimmer, T., Tahmasian, M., Yakushev, I., Sorg, C., et al. (2014). In Alzheimer's disease, hypometabolism in low-amyloid brain regions may be a functional consequence of pathologies in connected brain regions. Brain Connect. 4, 371-383. doi: 10.1089/brain.2013.0212

Konrad, K., and Eickhoff, S. B. (2010). Is the ADHD brain wired differently? A review on structural and functional connectivity in attention deficit hyperactivity disorder. Hum. Brain Mapp. 31, 904-916. doi: 10.1002/hbm. 21058

Lacoste, B., Comin, C. H., Ben-Zvi, A., Kaeser, P. S., Xu, X., Costa Lda, F., et al. (2014). Sensory-related neural activity regulates the structure of vascular networks in the cerebral cortex. Neuron 83, 1117-1130. doi: 10.1016/j.neuron. 2014.07.034

Lambert, J. C., Ibrahim-Verbaas, C. A., Harold, D., Naj, A. C., Sims, R., Bellenguez, C., et al. (2013). Meta-analysis of 74,046 individuals identifies 11 new susceptibility loci for Alzheimer's disease. Nat. Genet. 45, 1452-1458. doi: $10.1038 /$ ng.2802

LeBlanc, A. C. (2013). Caspase-6 as a novel early target in the treatment of Alzheimer's disease. Eur. J. Neurosci. 37, 2005-2018. doi: 10.1111/ejn.12250

LeBlanc, A., Liu, H., Goodyer, C., Bergeron, C., and Hammond, J. (1999). Caspase6 role in apoptosis of human neurons, amyloidogenesis and Alzheimer's disease. J. Biol. Chem. 274, 23426-23436. doi: 10.1074/jbc.274.33.23426

Leiserson, M. D. M., Vandin, F., Wu, H.-T., Dobson, J. R., Eldridge, J. V., Thomas, J. L., et al. (2015). Pan-cancer network analysis identifies combinations of rare somatic mutations across pathways and protein complexes. Nat. Genet. 47, 106-114. doi: 10.1038/ng.3168

Leventhal, G. E., Hill, A. L., Nowak, M. A., and Bonhoeffer, S. (2015). Evolution and emergence of infectious diseases in theoretical and real-world networks. Nat. Commun. 6:6101. doi: 10.1038/ncomms7101

Lloyd-Smith, J. O., Schreiber, S. J., Kopp, P. E., and Getz, W. M. (2005). Superspreading and the effect of individual variation on disease emergence. Nature 438, 355-359. doi: 10.1038/nature04153

Lull, M. E., and Block, M. L. (2010). Microglial activation and chronic neurodegeneration. Neurotherapeutics 7, 354-365. doi: 10.1016/j.nurt.2010. 05.014

Melie-García, L., Sanabria-Diaz, G., and Sánchez-Catasús, C. (2013). Studying the topological organization of the cerebral blood flow fluctuations in resting state. Neuroimage 64, 173-184. doi: 10.1016/j.neuroimage.2012.08.082

Messé, A., Rudrauf, D., Giron, A., and Marrelec, G. (2015). Predicting functional connectivity from structural connectivity via computational models using MRI: an extensive comparison study. Neuroimage 111, 65-75. doi: 10.1016/j. neuroimage.2015.02.001

Meyer-Lindenberg, A., and Tost, H. (2012). Neural mechanisms of social risk for psychiatric disorders. Nat. Neurosci. 15, 663-668. doi: 10.1038/nn.3083 
Montagne, A., Barnes, S. R., Sweeney, M. D., Halliday, M. R., Sagare, A. P., Zhao, Z., et al. (2015). Blood-brain barrier breakdown in the aging human hippocampus. Neuron 85, 296-302. doi: 10.1016/j.neuron.2014.12.032

Narayanan, M., Huynh, J. L., Wang, K., Yang, X., Yoo, S., McElwee, J., et al. (2014). Common dysregulation network in the human prefrontal cortex underlies two neurodegenerative diseases. Mol. Syst. Biol. 10:743. doi: 10.15252/msb. 20145304

Nath, S., Agholme, L., Kurudenkandy, F. R., Granseth, B., Marcusson, J., and Hallbeck, M. (2012). Spreading of neurodegenerative pathology via neuronto-neuron transmission of $\beta$-amyloid. J. Neurosci. 32, 8767-8777. doi: 10 . 1523/JNEUROSCI.0615-12.2012

Neumann, M., Sampathu, D. M., Kwong, L. K., Truax, A. C., Micsenyi, M. C., Chou, T. T., et al. (2006). Ubiquitinated TDP-43 in frontotemporal lobar degeneration and amyotrophic lateral sclerosis. Science 314, 130-133. doi: 10. $1126 /$ science. 1134108

Newman, M. E. (2002). Spread of epidemic disease on networks. Phys. Rev. E Stat. Nonlin. Soft Matter Phys. 66:016128. doi: 10.1103/physreve.66.016128

Nicolia, V., Lucarelli, M., and Fuso, A. (2014). Environment, epigenetics and neurodegeneration: focus on nutrition in Alzheimer's disease. Exp. Gerontol. doi: 10.1016/j.exger.2014.10.006. [Epub ahead of print].

O’Driscoll, L. (2011). Gene Expression Profiling: Methods and Protocols. 2nd Edn. Springer, Humana Press.

Pievani, M., Filippini, N., van den Heuvel, M. P., Cappa, S. F., and Frisoni, G. B. (2014). Brain connectivity in neurodegenerative diseases-from phenotype to proteinopathy. Nat. Rev. Neurol. 10, 620-633. doi: 10.1038/nrneurol. 2014.178

Proctor, C. J., Boche, D., Gray, D. A., and Nicoll, J. A. R. (2013). Investigating interventions in Alzheimer's disease with computer simulation models. PLoS One 8:e73631. doi: 10.1371/journal.pone.0073631

Qosa, H., Abuasal, B. S., Romero, I. A., Weksler, B., Couraud, P.-O., Keller, J. N., et al. (2014). Differences in amyloid- $\beta$ clearance across mouse and human blood-brain barrier models: kinetic analysis and mechanistic modeling. Neuropharmacology 79, 668-678. doi: 10.1016/j.neuropharm.2014.01.023

Quaegebeur, A., Lange, C., and Carmeliet, P. (2011). The neurovascular link in health and disease: molecular mechanisms and therapeutic implications. Neuron 71, 406-424. doi: 10.1016/j.neuron.2011.07.013

Raj, A., Kuceyeski, A., and Weiner, M. (2012). A network diffusion model of disease progression in dementia. Neuron 73, 1204-1215. doi: 10.1016/j.neuron. 2011.12.040

Ramanan, V. K., Risacher, S. L., Nho, K., Kim, S., Swaminathan, S., Shen, L., et al. (2014). APOE and BCHE as modulators of cerebral amyloid deposition: a florbetapir PET genome-wide association study. Mol. Psychiatry 19, 351-357. doi: $10.1038 / \mathrm{mp} .2013 .19$

Reynolds, J. J., and Stewart, G. S. (2013). A single strand that links multiple neuropathologies in human disease. Brain 136, 14-27. doi: 10. 1093/brain/aws310

Robberecht, W., and Philips, T. (2013). The changing scene of amyotrophic lateral sclerosis. Nat. Rev. Neurosci. 14, 248-264. doi: 10.1038/nrn3430

Roth, G. S., Lane, M. A., Ingram, D. K., Mattison, J. A., Elahi, D., Tobin, J. D., et al. (2002). Biomarkers of caloric restriction may predict longevity in humans. Science 297:811. doi: 10.1126/science.1071851

Sanz-Leon, P., Knock, S. A., Spiegler, A., and Jirsa, V. K. (2015). Mathematical framework for large-scale brain network modelling in the virtual brain. Neuroimage 111, 385-430. doi: 10.1016/j.neuroimage.2015.01.002

Sanz-Leon, P., Knock, S. A., Woodman, M. M., Domide, L., Mersmann, J., McIntosh, A. R., et al. (2013). The virtual brain: a simulator of primate brain network dynamics. Front. Neuroinform. 7:10. doi: 10.3389/fninf.2013.00010

Schmidt, R., de Reus, M. A., Scholtens, L. H., van den Berg, L. H., and van den Heuvel, M. P. (2015). Simulating disease propagation across white matter connectome reveals anatomical substrate for neuropathology staging in amyotrophic lateral sclerosis. Neuroimage doi: 10.1016/j.neuroimage.2015.04. 005. [Epub ahead of print].

Schwarz, Q., Gu, C., Fujisawa, H., Sabelko, K., Gertsenstein, M., Nagy, A., et al. (2004). Vascular endothelial growth factor controls neuronal migration and cooperates with Sema3A to pattern distinct compartments of the facial nerve. Genes Dev. 18, 2822-2834. doi: 10.1101/gad.322904
Scremin, O. (2012). The Human Nervous System-Cerebral Vascular System. Chapter 39, 3rd Edn. eds J. K. Mai and G. Paxinos (Academic Press), 1351-1374. doi: 10.1016/B978-0-12-374236-0.10039-2

Seeley, W. W., Crawford, R. K., Zhou, J., Miller, B. L., and Greicius, M. D. (2009). Neurodegenerative diseases target large-scale human brain networks. Neuron 62, 42-52. doi: 10.1016/j.neuron.2009.03.024

Sezgin, Z., and Dincer, Y. (2014). Alzheimer's disease and epigenetic diet. Neurochem. Int. 78, 105-116. doi: 10.1016/j.neuint.2014.09.012

Song, H.-L., Shim, S., Kim, D.-H., Won, S.-H., Joo, S., Kim, S., et al. (2014). $\beta$-Amyloid is transmitted via neuronal connections along axonal membranes. Ann. Neurol. 75, 88-97. doi: 10.1002/ana.24029

Sotero, R. C., Trujillo-Barreto, N. J., Iturria-Medina, Y., Carbonell, F., and Jimenez, J. C. (2007). Realistically coupled neural mass models can generate EEG rhythms. Neural Comput. 19, 478-512. doi: 10.1162/neco.2007.19.2.478

Soto, C., and Castilla, J. (2004). The controversial protein-only hypothesis of prion propagation. Nat. Med. 10(Suppl.), S63-S67. doi: 10.1038/nm1069

Sporns, O. (2011). The human connectome: a complex network. Ann. N Y Acad. Sci. 1224, 109-125. doi: 10.1111/j.1749-6632.2010.05888.x

Stam, C. J., and van Dijk, B. W. (2002). Synchronization likelihood: an unbiased measure of generalized synchronization in multivariate data sets. Physica D 163, 236-251. doi: 10.1016/s0167-2789(01)00386-4

Stice, E., Burger, K., and Yokum, S. (2013). Caloric deprivation increases responsivity of attention and reward brain regions to intake, anticipated intake, and images of palatable foods. Neuroimage 67, 322-330. doi: 10.1016/j. neuroimage.2012.11.028

Streit, W. J., Mrak, R. E., and Griffin, W. S. T. (2004). Microglia and neuroinflammation: a pathological perspective. J. Neuroinflammation 1:14 doi: 10.1186/1742-2094-1-14

Valdes-Sosa, P. A., Sanchez-Bornot, J. M., Sotero, R. C., Iturria-Medina, Y., Aleman-Gomez, Y., Bosch-Bayard, J., et al. (2009). Model driven EEG/fMRI fusion of brain oscillations. Hum. Brain Mapp. 30, 2701-2721. doi: 10 1002/hbm.20704

Waters, J. (2010). The concentration of soluble extracellular amyloid- $\beta$ protein in acute brain slices from CRND8 mice. PLoS One 5:e15709. doi: 10.1371/journal. pone.0015709

Wolf, L., Goldberg, C., Manor, N., Sharan, R., and Ruppin, E. (2011a). Gene expression in the rodent brain is associated with its regional connectivity. PLoS Comput. Biol. 7:e1002040. doi: 10.1371/journal.pcbi.1002040

Wolf, R. C., Grön, G., Sambataro, F., Vasic, N., Wolf, N. D., Thomann, P. A., et al. (2011b). Magnetic resonance perfusion imaging of resting-state cerebral blood flow in preclinical Huntington's disease. J. Cereb. Blood Flow Metab. 31, 1908-1918. doi: 10.1038/jcbfm.2011.60

Wooten, G. F., Currie, L. J., Bovbjerg, V. E., Lee, J. K., and Patrie, J. (2004). Are men at greater risk for Parkinson's disease than women? J. Neurol. Neurosurg. Psychiatry 75, 637-639. doi: 10.1136/jnnp.2003.020982

Zacchigna, S., Lambrechts, D., and Carmeliet, P. (2008). Neurovascular signalling defects in neurodegeneration. Nat. Rev. Neurosci. 9, 169-181. doi: 10.1038/ nrn2336

Zhang, B., Gaiteri, C., Bodea, L.-G., Wang, Z., McElwee, J., Podtelezhnikov, A., et al. (2013). Integrated systems approach identifies genetic nodes and networks in late-onset alzheimer's disease. Cell 153, 707-720. doi: 10.1016/j.cell.2013. 03.030

Zhou, J., Gennatas, E., Kramer, J., Miller, B., and Seeley, W. (2012). Predicting regional neurodegeneration from the healthy brain functional connectome. Neuron 73, 1216-1227. doi: 10.1016/j.neuron.2012.03.004

Conflict of Interest Statement: The authors declare that the research was conducted in the absence of any commercial or financial relationships that could be construed as a potential conflict of interest.

Copyright (C) 2015 Iturria-Medina and Evans. This is an open-access article distributed under the terms of the Creative Commons Attribution License (CC BY). The use, distribution and reproduction in other forums is permitted, provided the original author(s) or licensor are credited and that the original publication in this journal is cited, in accordance with accepted academic practice. No use, distribution or reproduction is permitted which does not comply with these terms. 